\title{
Trend of traumatic crown injuries and associated factors in preschool children
}

\section{Bernardo Antonio AGOSTINI (a) Lisara Tosatto PINTO(a) Mariangélica KOEHLER ${ }^{(b)}$ Bruno EMMANUELLI(a) Chaiana PIOVESAN(c) Thiago Machado ARDENGH(d)}

(a) Unviersidade Federal de Santa Maria - UFSM, Program in Dental Science, Santa Maria, RS, Brazil.

(b) Universidade Federal de Santa Maria - UFSM, Faculty of Dentistry, Santa Maria, RS, Brazil.

(c)Faculdade São Lucas, Dentistry, Porto Velho, RO, Brazil.

(d) Unviersidade Federal de Santa Maria - UFSM, Faculty of Dentistry, Department of Stomatology, Santa Maria, RS, Brazil.
Declaration of Interests: The authors certify that they have no commercial or associative interest that represents a conflict of interest in connection with the manuscript.

\section{Corresponding Author:}

Thiago Machado Ardenghi

E-mail: thiardenghi@hotmail.com

DOI: 10.1590/1807-3107BOR-2016.vol30.0112

Submitted: Sep 22, 2015

Accepted for publication: May 1, 2016

Last revision: Aug 8, 2016
Abstract: The aim of this study was to assess trends in traumatic crown injuries (TCIs), their prevalence, and association with socioeconomic and clinical factors in preschool children over a 5-year period. A series of cross-sectional surveys was conducted in Santa Maria, Brazil, on children attending a National Day of Children's Vaccination. The same protocol was used in all surveys conducted in 2008, 2010, and 2013. Clinical examinations were performed to evaluate types of traumatic crown injuries using the $\mathrm{O}^{\prime}$ brien index. A semi-structured questionnaire was answered by the parents. It provided information about several socioeconomic factors as well as the parents' perception of their child's oral health. The chi-square for trends test was used to verify changes in TCI prevalence throughout the years. The association between socioeconomic and clinical factors with TCI was assessed by the Poisson regression analysis. A total of 1,640 children were examined. The prevalence of TCIs decreased significantly from 2008 to $2013(10.12 \%$; $p$ 0.001). Central incisors were the most vulnerable to injury, independent of the year. Moreover, older children and those with inadequate lip coverage were more likely to present with some traumatic dental injury $(\mathrm{p}<0.001)$. prevalence of traumatic crown injuries decreased following the years, however TCI remain an important subject to consider when planning oral health policies.

Keywords: Child; Child, Preschool; Tooth Injuries.

\section{Introduction}

The prevalence of traumatic crown injuries (TCIs) has been evaluated in several countries; and the association with socioeconomic, clinical, and psychosocial indicators has been demonstrated in preschool children ${ }^{1,2,3,4,5,6,7,8}$. Data from the latest national population-based oral health study showed that $20.5 \%$ of children, aged 12 years, had TCI in at least one incisor 9 There is no national data for TCI prevalence in preschool children; however, regional studies reported a TCI prevalence ranging from 9.4\% to $41.6 \% 3,4,5,6,7,8,9,10$.

Studies conducted with Brazilian preschool children showed that TCI can produce a negative impact on the quality of life of children and their families ${ }^{11,12,13,14}$. Therefore, TCI is still considered a public dental problem due to high prevalence, impact on children's quality of life, and the high costs associated with treatment ${ }^{15,16}$. 
In this context, oral health surveys can provide important information for planning health strategies based on trends of oral health outcomes throughout a timeline. There is national data about trends of dental caries, as well as erosive tooth wear in children ${ }^{17,18}$. However, for TCI, these studies are scarce. There are few studies evaluating the trends in prevalence and severity of TCI in Brazilian preschool children. The results of these cross-sectional series indicating a significant increase in the prevalence of TCI suggests that public health efforts to reduce future TCI occurrence is needed ${ }^{19,20}$.

Recently, a systematic review on the prevalence and trends in dental trauma in permanent teeth in Latin America and the Caribbean was conducted with 24 studies. Most were conducted in Brazil, following a cross-sectional design with school-aged children. The authors suggest that there is no change in dental trauma over time. However, when considering Brazilian studies only (excluding those up to 2000s), the review showed a decrease in the prevalence of dental trauma ${ }^{21}$.

There is a lack of information about the occurrence of TCI over time as well as possible predictors for its occurrence. Therefore, assessing trends of TCI would provide important information for planning and developing public healthcare promotion, with an aim to reduce oral health inequalities. The aim of this study was to evaluate the trends in TCIs and their prevalence in Brazilian preschool children from 2008 to 2013. The association with socioeconomic and clinical factors was also assessed.

\section{Methodology}

This study used data from three oral health surveys administered to preschool children, aged 0-59 months, living in the city of Santa Maria, Brazil. Santa Maria is a medium-sized city with an estimated population of 261,031 inhabitants that includes 18,420 children, aged $0-5$ years ${ }^{22}$. The surveys were conducted using the same protocol during the National Day of Children's Vaccination in 2008, 2010, and 2013. Detailed information on the surveys has been previously described ${ }^{23,24,25,26}$.

In 2008, the sample calculation considered a standard error (SE) of 5\%, a confidence interval
(CI) level of $95 \%$, power of $80 \%$, design effect of 1.4 , and a TCI prevalence of $41.6 \%{ }^{9}$ (10\% for possible non-response rate is added). Therefore, the minimum sample size was estimated to be 410 children. In 2010, it was estimated that there was a minimum sample size of 512 participants, factoring in $95 \% \mathrm{CI}$, $4 \% \mathrm{SE}$, TCI prevalence of $31.7 \%$, design effect of 1.4 , and $10 \%$ for non-responders ${ }^{24}$. For the last survey performed in 2013, the same parameters were adopted excluding a previous prevalence of TCI, which was $13.3 \%$ (data from the survey conducted in 2010). After all procedures, the minimum sample size was 427 children.

Participants were systematically selected from all children who attended the National Day of Children's Vaccination in 15 health centers that are equally distributed in the five administrative regions of the city. The same health centers were used in all the surveys. The vaccination program has consistent uptake rates exceeding $97 \%$; and the selected health centers received almost $90 \%$ of the preschool children. Each fifth child in the queue in each health center in all the years was invited to participate. If the guardian declined, the next child in the queue was invited.

Data were collected through clinical oral examinations and structured interviews. Fifteen examiners and 30 assistants participated in the study each year. A benchmark dental examiner conducted the complete examiner training as well as the calibration process before each survey. In each year of data collection, different examiners and assistants conducted clinical examinations and interviews. All examiners reached satisfactory inter- and intra-examiner reliability. Kappa values for the presence of crown fractures were calculated on a tooth-by-tooth basis. The values for intraand inter-examiner agreement ranged between 0.75 and 0.91 . These values represent satisfactory intra-study reliability.

Children were individually examined while seated on a dental chair utilizing conventional dental illumination. Visual examinations were conducted with World Health Organization (WHO) periodontal probes and plane dental mirrors, using previously standardized criteria ${ }^{27}$. The clinical 
examination recorded information about TCIs using the same criteria employed in the United Kingdom Children's Dental Health Survey ${ }^{28}$ and also utilized in previous Brazilian studies ${ }^{24,29}$. It includes the following criteria: $0=$ no trauma; 1 = enamel fracture only; 2 = enamel-dentin fracture; 3 = enamel-dentin fracture with pulp exposure; 4 = signs of pulp involvement without signs of fracture; 5 = missing tooth due to TCI; or $6=$ other TCI. Lip coverage was considered adequate when the lips covered the anterior teeth completely in the at-rest position and inadequate if any part of the crown height was exposed and visible at same position ${ }^{30}$. Parents and guardians, through a structured questionnaire, relayed their socioeconomic characteristics. Information provided included characteristics of the children such as age, gender, skin, color, and household income. The questionnaire was applied in $10 \%$ of the sample previously to check its feasibility.

\section{Data analysis}

Data analyses were performed using STATA 12.0 software (Stata Corp. College Station, TX, USA). The prevalence of TCI was defined as the participants having at least one type of TCI according to the dental examination. A chi-square test for trends was used to compare the prevalence of TCI through the years. Multiple logistic regression analysis was performed to evaluate the association between the outcome (prevalence of TCI) and predictors. The Backward Stepwise method was used for including or excluding the variables from the final model. All variables with a $p<0.20$ on the unadjusted analysis were included in the fitting of the model; they were retained in the final model only if they presented at a p-value of $<0.05$ after the adjustment.

\section{Ethics}

This study was approved by the Municipal Government of Santa Maria-RS and the ethics in Research Committee of the Federal University of Santa Maria, protocol number - 0270.0.243.000-09. A signed and written informed consent was obtained from the parents of all children before the study was initiated.

\section{Results}

A total of 1,640 children were enrolled. We evaluated 455, 639, and 546 children in 2008, 2010, and 2013, respectively. Most of the children were white $(78.62 \%)$ and had adequate lip coverage (85.34\%). All other socio-demographic variables are summarized in Table 1. The TCI prevalence was 31.7\% in 2008, 13.3\% in 2010, and 22.5\% in 2013 (Figure 1). There was a significant decrease of $10.12 \%$ in the TCI prevalence between 2008 and 2013 ( $p=0.003$ ).

Regardless of the year, the maxillary central incisors were the most frequently traumatized teeth (Figure 2). The most common type of TCI were those involving enamel only (19.21\%), and less than $1 \%$ were injuries involving the pulp (Table 2). The prevalence of TCI was associated with age, lip coverage, and the survey's year. The prevalence increased with age and was higher in those without adequate lip coverage ( $\mathrm{PR}=1.51 ; 95 \% \mathrm{CI} 1.21-1.87)$. However, there was a decrease in the prevalence during the 5 years of study considering other possible confounding factors (Table 3).

\section{Discussion}

This study assessed the trends of TCI from 2008 to 2013 in a representative sample of preschool children from a medium-size city in Brazil. To the best of our knowledge, this is one of the few studies that evaluated the trends in TCI in primary teeth ${ }^{19,20,21}$.

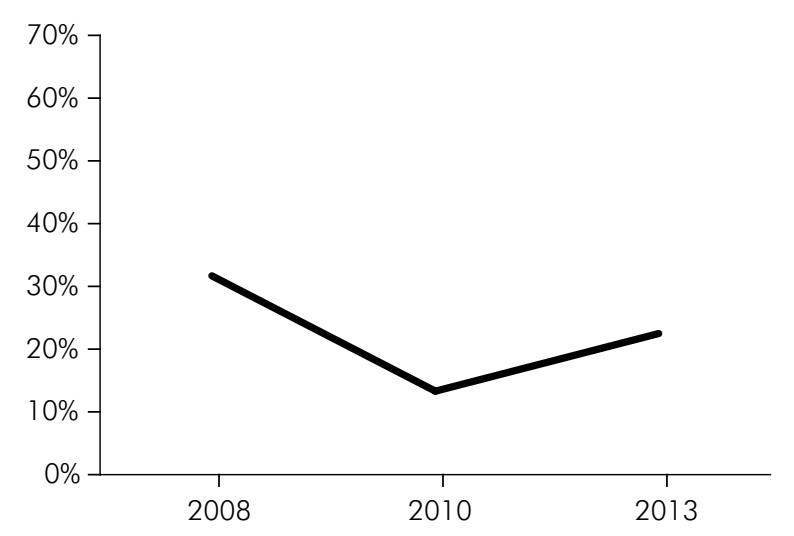

Figure 1. Trends in traumatic crown injuries prevalence between 2008 and 2013. Santa Maria, Brazil. $p=0.003$ according to chi-square for trend test. 
Table 1. Descriptive characteristics of the sample by year. Santa Maria, Brazil.

\begin{tabular}{|c|c|c|c|c|c|c|c|c|}
\hline \multirow{2}{*}{$\begin{array}{l}\text { Year } \\
\text { Variable }\end{array}$} & \multicolumn{2}{|c|}{2008} & \multicolumn{2}{|c|}{2010} & \multicolumn{2}{|c|}{2013} & \multicolumn{2}{|c|}{ Total } \\
\hline & $n$ & (\%) & $n$ & $(\%)$ & $n$ & (\%) & $n$ & $(\%)$ \\
\hline \multicolumn{9}{|l|}{ Gender } \\
\hline Male & 245 & 53.85 & 321 & 50.23 & 283 & 51.83 & 849 & 51.77 \\
\hline Female & 210 & 46.15 & 318 & 49.77 & 263 & 48.17 & 791 & 48.23 \\
\hline \multicolumn{9}{|l|}{ Age (months) } \\
\hline $0-23$ & 112 & 24.67 & 129 & 20.19 & 118 & 21.65 & 359 & 21.92 \\
\hline $24-35$ & 103 & 22.69 & 124 & 19.41 & 106 & 19.45 & 333 & 20.33 \\
\hline $36-47$ & 120 & 26.43 & 161 & 25.20 & 146 & 26.79 & 427 & 26.07 \\
\hline $48-60$ & 119 & 26.21 & 225 & 35.21 & 175 & 32.11 & 519 & 31.68 \\
\hline \multicolumn{9}{|l|}{ Skin Color } \\
\hline White & 345 & 75.82 & 501 & 78.40 & 441 & 81.22 & 1,287 & 78.62 \\
\hline Non-white & 110 & 24.18 & 138 & 21.60 & 102 & 18.78 & 350 & 21.38 \\
\hline \multicolumn{9}{|l|}{ Lip coverage } \\
\hline Adequate & 342 & 78.62 & 548 & 87.96 & 472 & 87.73 & 1,362 & 85.34 \\
\hline Inadequate & 93 & 21.38 & 75 & 12.04 & 66 & 12.27 & 234 & 14.66 \\
\hline \multicolumn{9}{|c|}{ Traumatic crown injuries } \\
\hline Without & 302 & 68.48 & 542 & 86.72 & 423 & 77.47 & 1.267 & 78.60 \\
\hline With & 139 & 31.52 & 83 & 13.28 & 123 & 22.53 & 345 & 21.40 \\
\hline \multicolumn{9}{|l|}{ Income* } \\
\hline$£$ Median & 241 & 54.28 & 212 & 35.27 & 264 & 49.81 & 717 & 45.52 \\
\hline$>$ Median & 203 & 45.72 & 389 & 64.73 & 266 & 50.19 & 858 & 54.48 \\
\hline
\end{tabular}

${ }^{*}$ cut-off point used was median of the corresponding year. Median in 2013 was approximately $R \$ 1,700 ; 2010$ was approximately R\$1,470; and 2008 was approximately $R \$ 1,020$.

TCIs in preschool children showed a significant decrease from 2008 to $2013(10.12 \%, p=0.003)$. These results are in accordance with a recent systematic review that assessed studies from Latin America and the Caribbean and demonstrated a relative stability in the prevalence of TCI throughout the past decade. However, when considering Brazilian studies up to the 2000 s, a trend of decrease in the prevalence of dental trauma could be observed for the permanent dentition. Although the systematic review included only studies with permanent teeth, we were able to compare our results. Other studies included in the review used the same criteria for TCI presence and were conducted in similar regions. The authors reported that the decrease could be due to more intense, preventive efforts taking place in the south and southeast regions of Brazil, the richest areas of the country ${ }^{21}$.

However, our results disagree with some regional trend studies conducted in a Brazilian southeastern city. Bonini et al. in 2009 reported a significant increase $(p=0.002)$ between 2002 and $2006^{19}$.

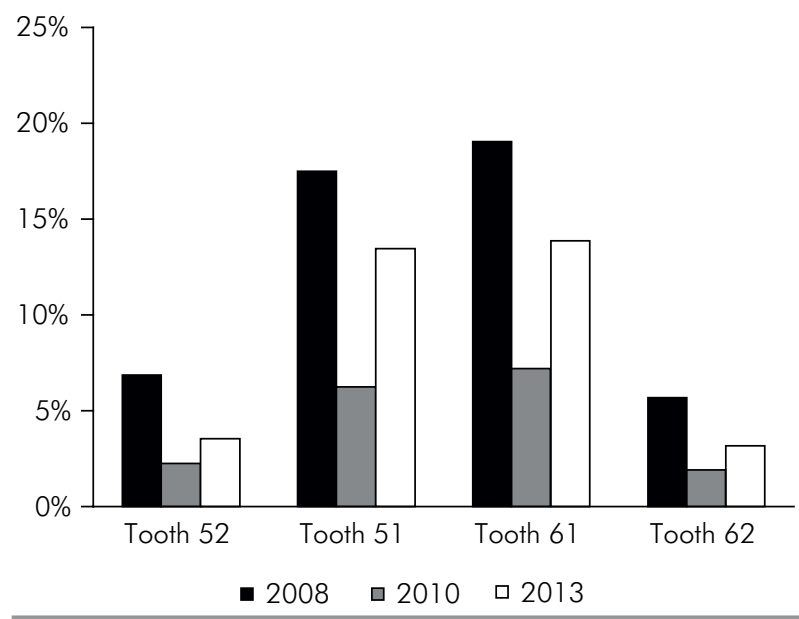

Figure 2. Prevalence of traumatic crown injuries in each year according the teeth.

Recently, a subsequent study conducted in the same population reported an increase of TCI prevalence in a representative sample of preschool children located in the Brazilian city of Diadema between 2002 and $2012^{20}$. The studies used different criteria other than 
Table 2. Prevalence of each type of traumatic crown injuries by year. Santa Maria, Brazil.

\begin{tabular}{|c|c|c|c|c|c|c|c|c|c|}
\hline Year & 2008 & $=442)$ & 201 & $=625)$ & 201 & $=531)$ & Total ( $r$ & 1,598) & $\mathrm{P}^{*}$ \\
\hline Type of trauma* & $\mathrm{n}$ & $(\%)$ & $\mathrm{n}$ & $(\%)$ & $\mathrm{n}$ & (\%) & $\mathrm{n}$ & $(\%)$ & $r$ \\
\hline Enamel fractures only & 142 & 32.13 & 62 & 9.92 & 103 & 19.40 & 307 & 19.21 & $<0.01$ \\
\hline Enamel-Dentin fracture & 6 & 1.36 & 16 & 2.56 & 17 & 3.20 & 39 & 2.44 & 0.17 \\
\hline Enamel-Dentin fracture with Pulp exposure & 7 & 1.58 & 2 & 0.32 & 5 & 0.94 & 14 & 0.88 & 0.09 \\
\hline $\begin{array}{l}\text { Signs of pulp involvement without signs of } \\
\text { fracture }\end{array}$ & 6 & 1.36 & 4 & 0.64 & 8 & 1.51 & 18 & 1.13 & 0.32 \\
\hline Missing tooth due to $\mathrm{TCl}$ & 6 & 1.36 & 2 & 0.32 & 5 & 0.94 & 13 & 0.81 & 0.16 \\
\hline Other $\mathrm{TCl}$ & - & - & 1 & 0.16 & 1 & 0.19 & 2 & 0.12 & 0.39 \\
\hline
\end{tabular}

TDI: traumatic dental injuries; *according Chi-square for trends test.

Table 3. Poisson regression of traumatic crown injuries and associated factors. Santa Maria, Brazil.

\begin{tabular}{|c|c|c|c|c|}
\hline \multirow{2}{*}{ Variable } & \multicolumn{4}{|c|}{ Traumatic crown injuries } \\
\hline & PR crude $(95 \% \mathrm{Cl})$ & $\mathrm{p}$ & PR adj. $(95 \% \mathrm{Cl})$ & $p$ \\
\hline \multicolumn{5}{|l|}{ Year } \\
\hline 2008 & 1.00 & - & - & - \\
\hline 2010 & $0.42(0.33-0.53)$ & $<0.001$ & $0.43(0.33-0.56)$ & $<0.001$ \\
\hline 2013 & $0.71(0.58-0.88)$ & 0.003 & $0.72(0.58-0.90)$ & $<0.001$ \\
\hline \multicolumn{5}{|l|}{ Age (months) } \\
\hline $0-23$ & 1.00 & - & - & - \\
\hline $24-35$ & $1.99(1.39-2.85)$ & $<0.001$ & $1.90(1.32-2.72)$ & $<0.001$ \\
\hline $36-47$ & $2.13(1.51-3.00)$ & $<0.001$ & $2.07(1.47-2.91)$ & $<0.001$ \\
\hline $48-59$ & $2.20(1.57-3.08)$ & $<0.001$ & $2.31(1.66-3.23)$ & $<0.001$ \\
\hline \multicolumn{5}{|l|}{ Gender $^{+}$} \\
\hline Male & 1.00 & - & - & - \\
\hline Female & $0.88(0.73-1.06)$ & 0.196 & - & - \\
\hline \multicolumn{5}{|l|}{ Skin Color ${ }^{+}$} \\
\hline White & 1.00 & - & - & - \\
\hline Non-White & $1.02(0.81-1.28)$ & 0.854 & - & - \\
\hline \multicolumn{5}{|l|}{ Income $e^{*+}$} \\
\hline$\leq$ Median & 1.00 & - & - & - \\
\hline$>$ Median & $0.83(0.69-1.01)$ & 0.061 & - & - \\
\hline \multicolumn{5}{|l|}{ Lip coverage } \\
\hline Adequate & 1.00 & - & - & - \\
\hline Inadequate & $1.68(1.35-2.08)$ & $<0.001$ & $1.51(1.21-1.87)$ & $<0.001$ \\
\hline
\end{tabular}

the TCI diagnosis used in our study. Moreover, these were performed in different geographic regions of the country. Different cultural aspects could be the reasons for such differences. In 2004, the current public dental policy "Smiling Brazil" was created, another possible contributor. This set of actions with a goal of improving the population's oral health includes everything from prevention to complex rehabilitation $^{31}$. Hence, the difference between the trends studies could be due to these improvements in public health in recent years because they represent different regions of the country.

The decrease presented in TCI prevalence in this study could be explained by the hypothesis that children are currently adopting more sedentary lifestyles $^{32,33}$ as well as seeking early treatment for 
malocclusion. According to one recent study, the traditional child activity games are being replaced by other behaviors during leisure time, including watching TV and using computers, videogames, or other electronic entertainment ${ }^{33}$. Furthermore, a study conducted in South Africa also reported that preschool children are spending more time in sedentary behavior than engaging in moderate-to-vigorous physical activity ${ }^{34}$. Sedentary behavior could be considered a protection for the development of TCI. However, we did not assess sedentary habits in our study, and further studies are necessary to assess such a hypothesis with evidence.

Furthermore, the decrease in the prevalence must be considered carefully because there was an increase from 2010 to 2013 (9.2\%) in our study. This increase and the short period of evaluation did not allow for an accurate trend shape. An explanation for this increase relies on information bias. Although the examiners were well trained and a standardized criterion was used, some diagnostic decisions may have changed in each year. Another possibility is that random errors occurred in the sampling process and in the short period of evaluation. However, a shorter period of evaluation was previously used ${ }^{19}$. It also important highlight that TCI trend studies are important since it could predict population behavior facing the outcome. Moreover, TCI impacts the quality of life of preschool children ${ }^{35}$.

In comparison to other studies, the majority of TCIs showed that the upper central incisors were more affected than the lateral incisors ${ }^{3,19,23}$. The maxillary central incisors are generally more inclined than the other teeth. They tend to be the first to receive a direct impact when the child falls, leading to a fracture in the enamel or dentin or causing major complications ${ }^{24}$. Crown fractures involving the enamel only were the most common type of injury in our sample (19.21\%), followed by those in the enamel and dentin (2.44\%). It is suggested that enamel fractures are the most common because most of the injuries are caused by a low intensity impact. The minor damage to the enamel and dentin should be assessed with caution; however, because an the seriousness of the injury could appear minor at the time of the accident, it may eventually result in pulp necrosis ${ }^{36}$. Nevertheless, it is interesting to emphasize that the treatment of TCIs tends to be neglected, which could affect a child's ability to eat, speak, and socialize ${ }^{19}$. It highlights the importance of relaying more widespread information and explanation about TCI treatment. Neglecting case resolution may result in difficult outcomes to both primary and permanent dentitions

Our study showed an association between age and TCI. Older children were more likely to present TCI than their counterparts, particularly in the age group of $48-59$ months $(\mathrm{PR}=2.20,95 \% \mathrm{CI}=1.57-3.08, \mathrm{p}<0.001)$. In accordance with one previous study, the injuries occur in this group because they are just beginning to move independently and explore their environment with a relative lack of coordination 7 . Another positive correlation was shown between lip coverage and TCI. Those children classified without adequate lip coverage were more likely to have traumatic injuries in maxillary incisors than those with adequate lip coverage. Our results are in accordance with previous studies ${ }^{3,8,37}$, emphasizing the importance of anatomic predisposing factors for TCI occurrence. TCIs occur approximately twice as frequently among children with protruding incisors compared with those in children with normal occlusion ${ }^{3}$. Furthermore, the authors of a systematic review suggested that lip coverage and early treatment of malocclusion are important variables to consider as part of promotion of public health policies ${ }^{21}$. We have not evaluated overjet, and this could explain part of the association. However, a previous study reported that overjet is associated with TCI only when inadequate lip coverage is also present ${ }^{38}$.

Some limitations must be considered. A possible underestimation of prevalence could have occurred because we used a criteria focused only on the visible signs of TCIs. Therefore, other types of TCI, such as concussion and luxation, were ignored. However, the criteria used in the surveys were consistent with previous studies, allowing for comparison ${ }^{24,35}$. Moreover, despite the examiners being calibrated in each survey, some small differences in diagnostic decisions may occur. It could happen because of the criteria used, which rely on visual diagnosis, excluding complementary exams. Some comparisons are not possible because there are no well-defined 
criteria to assess TCI. However, a recent systematic review showed that the index adopted in this study is one of the most common criteria used in Brazilian studies $^{21}$. Another limitation was the short period of evaluation; only three points in time were used to describe our results. Further assessments over a longer period may allow us to detect more reliable effects of population changes in the trends of TCI, thereby being able to predict a better trend shape.

In conclusion, this study showed that trends of TCI in preschoolers decreased significantly between 2008 and 2013. The results are important for health

\section{References}

1. Kahabuka FK, Plasschaert A, Hoft MV. Prevalence of teeth with untreated dental trauma among nursery and primary school pupils in Dar es Salaam, Tanzania. Dent Traumatol. 2001;17(3):109-13. doi:10.1034/j.1600-9657.2001.017003109.x

2. Granville-Garcia AF, Menezes VA, Lira PIC. Dental trauma and associated factors in Brazilian preschoolers. Dent Traumatol. 2006;22(6):318-22. doi:10.1111/j.1600-9657.2005.00390.x

3. Oliveira LB, Marcenes W, Ardenghi TM, Sheiham A, Bönecker M. Traumatic dental injuries and associated factors among Brazilian preschool children. Dent Traumatol. 2007;23(2):76-81. doi:10.1111/j.1600-9657.2005.00413.x

4. Ferreira JMS, Andrade EMF, Katz CRT, Rosenblatt A. Prevalence of dental trauma in deciduous teeth of Brazilian children. Dent Traumatol. 2009;25(2):219-23. doi:10.1111/j.1600-9657.2008.00754.x

5. Robson F, Ramos-Jorge ML, Bendo CB, Vale MP, Paiva SM, Pordeus IA. Prevalence and determining factors of traumatic injuries to primary teeth in preschool children. Dent Traumatol. 2009;25(1):118-22. doi:10.1111/j.1600-9657.2008.00725.x

6. Feldens CA, Kramer PF, Ferreira SH, Spiguel MH, Marquezan M. Exploring factors associated with traumatic dental injuries in preschool children: a Poisson regression analysis. Dent Traumatol. 2010;26(2):143-8. doi:10.1111/j.1600-9657.2009.00858.x

7. Rhouma O, McMahon AD, Conway DI, Armstrong M, Welbury R, Goodall C. Facial injuries in Scotland 2001-2009: epidemiological and sociodemographic determinants. Br J Oral Maxillofac Surg. 2013;51(3):211-6. doi:10.1016/j.bjoms.2012.04.267

8. Goettems ML, Torriani DD, Hallal PC, Correa MB, Demarco FF. Dental trauma: prevalence and risk factors in schoolchildren. Community Dent Oral Epidemiol. 2014;42(6):581-90. doi:10.1111/cdoe.12113 sectors to implement health promotion strategies for the management of these injuries in early ages.

\section{Acknowledgements}

We thank the Coordenação de Apoio e Pesquisa do Ensino Superior (CAPES) that provided scholarship to some students involved in this project. We also thank the Conselho Nacional de Desenvolvimento Científico e Tecnológico (CNPq - process 477118/2013-5 and CNPq process 308141/2012-1) for supporting this epidemiological research. We are grateful to all families that agreed to participate in this research.

9. Ministério da Saúde (BR), Secretaria de Vigilância em Saúde, Departamento de Atenção Básica. Projeto SBBrasil 2010: resultados principais. Brasília, DF: Ministério da Saúde; 2011.

10. Jorge KO, Moysés SJ, Ferreira EF, Ramos-Jorge ML, Zarzar PMPA. Prevalence and factors associated to dental trauma in infants 1-3 years of age. Dent Traumatol. 2009;25(2):185-9. doi:10.1111/j.1600-9657.2008.00730.x

11. Kramer PF, Feldens CA, Ferreira SH, Bervian J, Rodrigues PH, Peres MA. Exploring the impact of oral diseases and disorders on quality of life of preschool children. Community Dent Oral Epidemiol. 2013;41(4):327-35. doi:10.1111/cdoe.12035

12. Viegas C, Scarpelli AC, Carvalho AC, Ferreira FM, Pordeus IA, Paiva SM. Impact of traumatic dental injury on quality of life among Brazilian preschool children and their families. Pediatr Dent. 2012;34(4):300-6.

13. Aldrigui JM, Abanto J, Carvalho TS, Mendes FM, Wanderley MT, Bönecker M et al. Impact of traumatic dental injuries and malocclusions on quality of life of young children. Health Qual Life Outcomes. 2011;9(1):78. doi:10.1186/1477-7525-9-78

14. Viegas CM, Paiva SM, Carvalho AC, Scarpelli AC, Ferreira FM, Pordeus IA. Influence of traumatic dental injury on quality of life of Brazilian preschool children and their families. Dent Traumatol. 2014;30(5):338-47. doi:10.1111/edt.12091

15. Glendor U. Epidemiology of traumatic dental injuries: a 12 year review of the literature. Dent Traumatol. 2008;24(6):603-11. doi:10.1111/j.1600-9657.2008.00696.x

16. Rhouma O, McMahon AD, Welbury R. Traumatic dental injury and social deprivation in five-year-old children in Scotland 1993-2007. Br Dent J; 2013;214(10):E26. doi:10.1038/sj.bdj.2013.505

17. Bönecker M, Ardenghi TM, Oliveira LB, Sheiham A, Marcenes $\mathrm{W}$. Trends in dental caries in 1- to 4-year-old children in a Brazilian city between 1997 and 2008. Int J Paediatr Dent. 2010;20(2):125-31. doi:10.1111/j.1365-263X.2009.01030.x 
18. Murakami C, Tello G, Abanto J, Oliveira LB, Bonini GC, Bönecker M. Trends in the prevalence of erosive tooth wear in Brazilian preschool children. Int J Paediatr Dent. 2016;26(1):60-5. doi:10.1111/ipd.12159

19. Bonini GAVC, Marcenes W, Oliveira LB, Sheiham A, Bönecker M. Trends in the prevalence of traumatic dental injuries in Brazilian preschool children. Dent Traumatol. 2009;25(6):594-8. doi:10.1111/j.1600-9657.2009.00826.x

20. Tello G, Bonini GC, Murakami C, Abanto J, Oliveira LB, Bönecker $\mathrm{M}$. Trends in the prevalence of traumatic crown injuries and associated factors in Brazilian preschool children: 10-year observational data. Dent Traumatol. 2016;32(4):274-80. doi:10.1111/edt.12255

21. Aldrigui JM, Jabbar NS, Bonecker M, Braga MM, Wanderley MT. Trends and associated factors in prevalence of dental trauma in Latin America and Caribbean: a systematic review and meta-analysis. Community Dent Oral Epidemiol. 2014;42(1):30-42. doi:10.1111/cdoe.12053

22. Instituto Brasileiro de Geografia e Estatística - IBGE, Banco de Dados agregados. Sample results: work and income. Rio de Janeiro: Instituto Brasileiro de Geografia e Estatística; 2010 [cited 2013 May 22]. Available from: http://www.sidra.ibge.gov.br/bda/tabela/protabl

23. Piovesan C, Mendes FM, Ferreira F V, Guedes RS, Ardenghi TM. Socioeconomic inequalities in the distribution of dental caries in Brazilian preschool children. J Public Health Dent. 2010;70(4):319-26. doi:10.1111/j.1752-7325.2010.00191.x

24. Piovesan C, Guedes RS, Casagrande L, Ardenghi

TM. Socioeconomic and clinical factors associated with traumatic dental injuries in Brazilian preschool children. Braz Oral Res. 2012;26(5):464-70. doi:10.1590/S1806-83242012000500014

25. Machry RV, Tuchtenhagen S, Agostini BA, Teixeira CRS, Piovesan C, Mendes FM et al. Socioeconomic and psychosocial predictors of dental healthcare use among Brazilian preschool children. BMC Oral Health. 2013;13:60. doi:10.1186/1472-6831-13-60

26. Agostini BA, Machry RV, Teixeira CR, Piovesan C, Oliveira MD, Bresolin CR et al. Self-perceived oral health influences tooth brushing in preschool children. Braz Dent J. 2014;25(3):248-52. doi:10.1590/0103-6440201302426

27. World Health Organization - WHO. Oral health surveys, basic methods. Geneva: World Health Organization; 1997.

28. O'Brien M. Children's dental health in the United Kingdom 1993. London: HMSO; 1994.
29. Damé-Teixeira N, Alves LS, Ardenghi TM, Susin C, Maltz M. Traumatic dental injury with treatment needs negatively affects the quality of life of Brazilian schoolchildren. Int J Paediatr Dent. 2013;23(4):266-73. doi:10.1111/ipd.12002

30. O'Mullane DM. Injured permanent incisor teeth: an epidemiological study. J Ir Dent Assoc 1972;18(4):160-73.

31. Pucca Jr GA., Gabriel M, Araujo ME, Almeida FCS. Ten years of a national oral health policy in brazil : innovation, boldness, and numerous challenges. J Dental Res. 2015;94(10):1333-7. doi:10.1177/0022034515599979

32. Dollman J, Norton K, Norton L. Evidence for secular trends in children's physical activity behaviour. Br J Sports Med. 2005;39(12):892-7. doi:10.1136/bjsm.2004.016675

33. Santos FK, Maia JR, Gomes TNQF, Daca T, Madeira A, Damasceno A et al. Secular trends in habitual physical activities of Mozambican children and adolescents from Maputo city. Int J Environ Res Public Health. 2014;11(10):10940-50. doi:10.3390/ijerph111010940

34. Jones S, Hendricks S, Draper CE. Assessment of physical activity and sedentary behavior at preschools in Cape Town, South Africa. Child Obes. 2014;10(6):501-10. doi:10.1089/chi.2014.0097

35. Abanto J, Tello G, Bonini GC, Oliveira LB, Murakami C, Bönecker M. Impact of traumatic dental injuries and malocclusions on quality of life of preschool children: a population-based study. Int J Paediatr Dent. 2015;25(1):18-28. doi:10.1111/ipd.12092

36. Marcenes W, Zabot NE, Traebert J. Socio-economic correlates of traumatic injuries to the permanent incisors in schoolchildren aged 12 years in Blumenau, Brazil. Dent Traumatol. 2001;17(5):222-6. doi:10.1034/j.1600-9657.2001.170507.x

37. Norton E, O'Connell AC. Traumatic dental injuries and their association with malocclusion in the primary dentition of Irish children. Dent Traumatol. 2012;28(1):81-6. doi:10.1111/j.1600-9657.2011.01032.x

38. Bonini GC, Bönecker M, Braga MM, Mendes FM. Combined effect of anterior malocclusion and inadequate lip coverage on dental trauma in primary teeth. Dent Traumatol. 2012;28(6):437-40. doi:10.1111/j.1600-9657.2012.01117.x 